Ganbar A. Guluyev ', Fahrad H. Pashayev ${ }^{2}$,

Bikas S. Agayev ${ }^{3}$, Ulkar E. Sattarova ${ }^{4}$, Vusal V. Bayramov ${ }^{5}$

DOI: 10.25045/jpit.v09.i2.03

${ }^{1}$ Institute of Control Systems of ANAS, Baku, Azerbaijan, scb_06@gmail.com,

${ }^{2}$ Institute of Control Systems of ANAS, Baku, Azerbaijan, pasha.farhad@gmail.com,

${ }^{3}$ Institute of Information Technology of ANAS, Baku, Azerbaijan, bikies418@gmail.com,

${ }^{4}$ Azerbaijan University of Architecture and Construction, Baku, Azerbaijan,

ulker.rzaeva@gmail.com,

${ }^{5}$ Institute of Control Systems of ANAS, Baku, Azerbaijan, vusal_bayramov84@mail.ru

\title{
A WAVELET-BASED FILTRATION METHOD OF NOISY TECHNOLOGICAL ACOUSTIC AND SPEECH SIGNALS
}

In this paper a wavelet-based denoising algorithm applied to technological acoustic and speech noisy signals is proposed. The analysis is done by applying discrete wavelet transform to technological acoustic and speech noisy signals and applying new denoising method for reconstruction of wavelets. The analysis is verified through simulation studies.

Keywords: Wavelet Transform, Acoustic Signals, Speech Signals, Denoising, MATLAB.

\section{Introduction}

In order to eliminate noise within the passband of the signal, various denoising algorithms have been proposed [1-5]. A comparative simulation study of wavelet based denoising algorithms is done in [6]. A novel denoising approach to photoacoustic signals is proposed in [7]. In this paper a new wavelet based denoising method of technological acoustic and speech signal processing is proposed. The proposed wavelet denoising method has the advantages of fast computing speed and a wide range of adaptability.

\section{A review of discrete wavelet transform and problem statement}

Traditionally the acoustic signals are analyzed in the frequency domain using Fourier transform. This technique, though providing interesting results, has major limitations due to the so-called time-frequency ambiguity. Large time windows produce better frequency resolution, but at the cost of a coarse time resolution (a problem for non-stationary signals), whereas the reverse is true for small moving windows. Therefore it is impossible to clearly resolve simultaneously high- and low-frequency events in a single analysis. Considering seismic signals are very complex and occur in a wide spectral range, this is a significant limitation. Discrete Wavelet Transform (DWT) offers the following advantages: (1) resolves the time-frequency ambiguity; (2) analyses multiple scales with suitable windows enhancing phenomena on each scale; (3) allows for the use of base functions different from sin/cosine waves, and (4) can be computed efficiently via a fast recursion of convolution decimation operations. The discrete wavelet transform is defined as:

$$
d_{j, n}=\left\langle x(t), \varphi_{j, n}(t)\right\rangle=\int_{-\infty}^{+\infty} x(t) \varphi_{j, n}(t) d t
$$

Where the mother wavelet function $\varphi_{j, n}(t)$ is defined as:

$$
\varphi_{j, n}(t)=a_{0}^{-j / 2} \varphi_{j, n}\left(a_{0}^{-j} t-n b_{0}\right)
$$

The scaling $a_{0}$ and translation $b_{0}$ parameters are specifically chosen such that $\varphi_{j, n}(t)$ constitute orthonormal bases with $a_{0}=2$ and $b_{0}=1$ [8-10]. So we have:

$$
\varphi_{j, n}(t)=2^{-j / 2} \varphi_{j, n}\left(2^{-j} t-n\right)
$$

Using equation (1), the orthonormal wavelet transform is thus given by: 


$$
d_{j, n}=\left\langle x(t), \varphi_{j, n}(t)\right\rangle=2^{-j / 2} \int_{-\infty}^{+\infty} x(t) \varphi_{j, n}\left(2^{-j} t-n\right) d t
$$

The inverse discrete wavelet transform is given by:

$$
x(t)=\sum_{j=1}^{J} \sum_{n=1}^{N} d_{j, n} \varphi_{j, n}(t)
$$

The discrete wavelet transform (DWT) is computed by successive lowpass and highpass filtering of the discrete time-domain signal as shown in fig. 1. This is called the Mallat algorithm or Mallat-tree decomposition. Its significance is in the manner it connects the continuous-time multiresolution to discrete-time filters. In the figure, the signal is denoted by the sequence $\mathrm{x}[\mathrm{n}]$, where $\mathrm{n}$ is an integer. The low pass filter is denoted by $\mathrm{G}_{0}$ while the high pass filter is denoted by $\mathrm{H}_{0}$. At each level, the high pass filter produces detail information, d[n], while the low pass filter associated with scaling function produces coarse approximations, a[n].

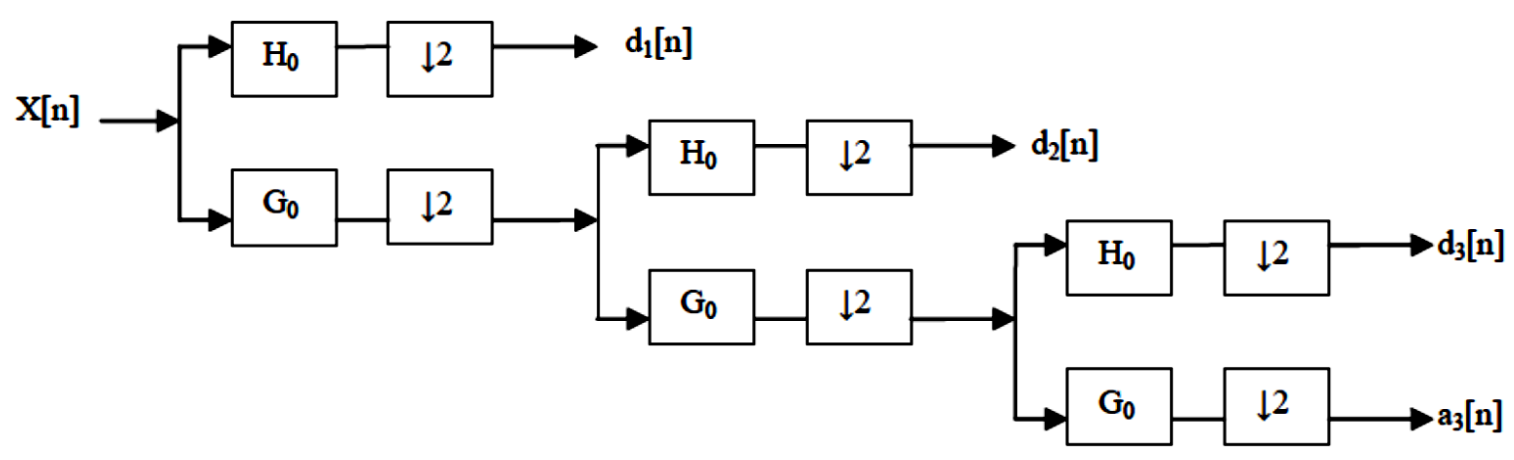

Fig. 1. Three-level wavelet decomposition tree

At each decomposition level, the half band filters produce signals spanning only half the frequency band. This doubles the frequency resolution as the uncertainty in frequency is reduced by half. In accordance with Nyquist's rule, if the original signal has a highest frequency of $\omega$, which requires a sampling frequency of $2 \omega$ radians, then it now has a highest frequency of $\omega / 2$ radians. It can now be sampled at a frequency of $\omega$ radians thus discarding half the samples with no loss of information. This decimation by 2 halves the time resolution as the entire signal is now represented by only half the number of samples. Thus, while the half band low pass filtering removes half of the frequencies and thus halves the resolution, the decimation by 2 doubles the scale. With this approach, the time resolution becomes arbitrarily good at high frequencies, while the frequency resolution becomes arbitrarily good at low frequencies. The filtering and decimation process is continued until the desired level is reached. The maximum number of levels depends on the length of the signal.

The DWT of the original signal is then obtained by concatenating all the coefficients, $a[n]$ and $d[n]$, starting from the last level of decomposition. Fig. 2 shows the reconstruction of the original signal from the wavelet coefficients. 


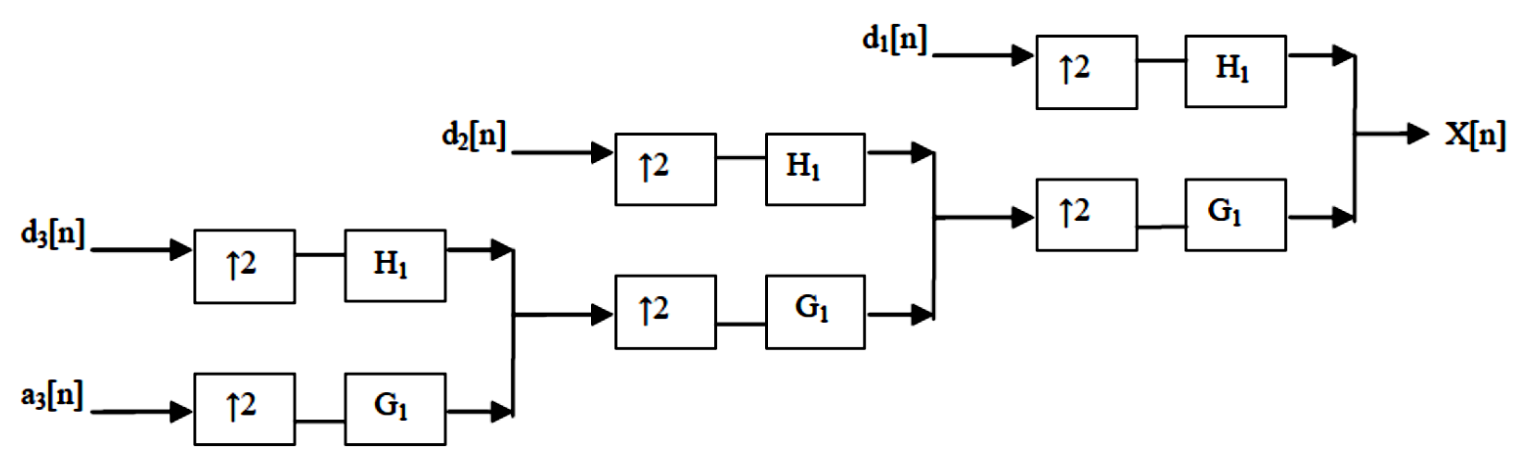

Fig. 2. Three-level wavelet reconstruction tree

Basically, the reconstruction is the reverse process of decomposition. The approximation and detail coefficients at every level are upsampled by two, passed through the low pass and high pass synthesis filters and then added. This process is continued through the same number of levels as in the decomposition process to obtain the original signal. The Mallat algorithm works equally well if the analysis filters, $G_{0}$ and $H_{0}$, are exchanged with the synthesis filters, $G_{1}$ and $H_{1}$.

The article provides a method of stimulating discrete Velvet transformations of the filtration of acoustic signals from the noise.

\section{The proposed method}

Wavelet transform has a concentrated ability and it can concentrate the energy of signal onto the wavelet coefficients [11]. The wavelet coefficients of signal are larger than the wavelet coefficients of the noise in wavelet transform domain. Therefore, we can use threshold method on the transform coefficients to remove noise. A seismic signal $x(t)$ can be modeled as the sum of a noise process $e(t)$ and the part of the signal that describes the seismic event $x^{\prime}(t)$ :

$$
x(t)=x^{\prime}(t)+e(t)
$$

Describing it in terms of wavelets coefficients $d_{j, n}$ we use:

$$
d_{j, n}(x(t))=d_{j, n}\left(x^{\prime}(t)\right)+d_{j, n}(e(t))
$$

Where $j$ is the scale and $n$ is the number of points in each scale. The goal is to minimize the contribution of the noise process $e(t)$. In the proposed denoising method of noisy seismic signals the orthonormal discrete wavelet transform is used on noisy signals and the appropriate wavelet as well as wavelet decomposition level $\mathrm{j}$ is selected. Then the wavelet decomposition on noisy signal to level $\mathrm{j}$ is carried out and the corresponding wavelet coefficients are obtained. The threshold quantification for wavelet coefficients under different decomposition scales is conducted. The following threshold is used:

$$
d_{j, n}^{\prime}= \begin{cases}\lambda-d_{j, n} & \left|d_{j, n}\right| \geq \lambda, d_{j, n} \geq 0 \\ d_{j, n}-\lambda & \text { otherwise }\end{cases}
$$

Where $\lambda$ is defined as follows:

$$
\lambda=\frac{\sum_{j=1}^{J} \sum_{n=1}^{N} d_{j, n}}{J \times N}
$$

The inverse discrete wavelet transform is carried out and the signal is reconstructed with new wavelet coefficients processed in equations (8) and (9). We have the following equation: 


$$
x^{\prime}(t)=\sum_{j=1}^{J} \sum_{n=1}^{N} d_{j, n}^{\prime} \varphi_{j, n}(t)
$$

The proposed wavelet denoising method has the advantages of fast computing speed and a wide range of adaptability. Therefore, this paper combines the wavelet transform method and reconstruction algorithm together to achieve the denoising process of noisy seismic signals. That is, first we can use the wavelet transform method to remove noise, and then apply the reconstruction algorithm to its output denoising.

\section{Simulation results}

According to the new proposed method described in the above section, the simulation is carried out on noisy technological acoustic and speech signals. The program has been written using the MATLAB software. Fig. 3 and Fig. 4 show the noisy signals (signal 1 and 2) and the denoising results using new proposed method simultaneously.

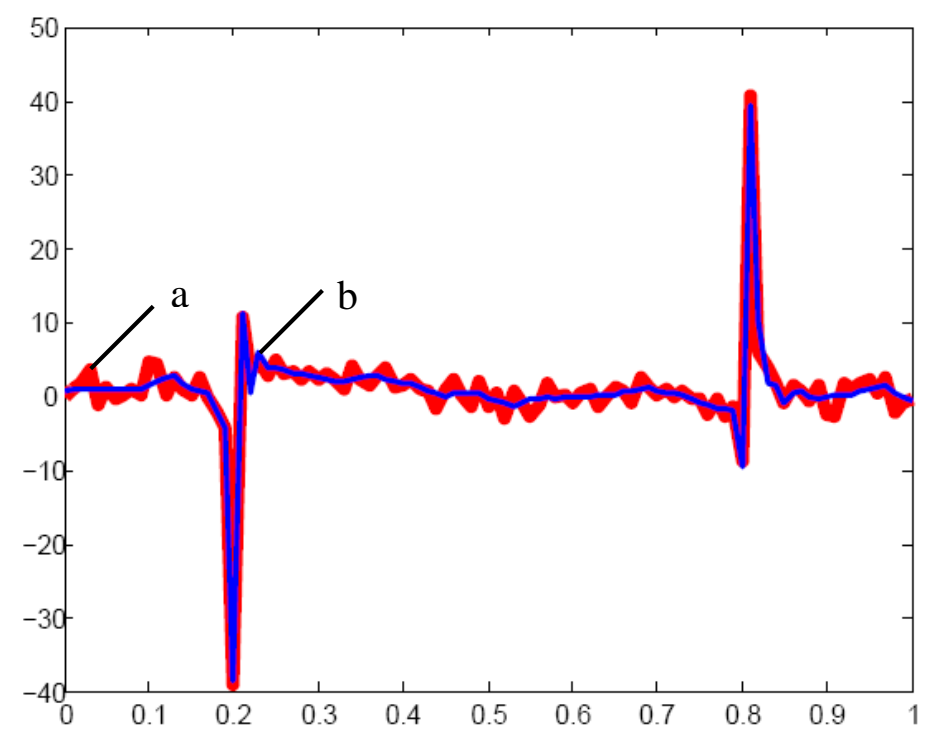

Fig. 3. Signal 1: noisy (red-a) and denoising (blue-b) result

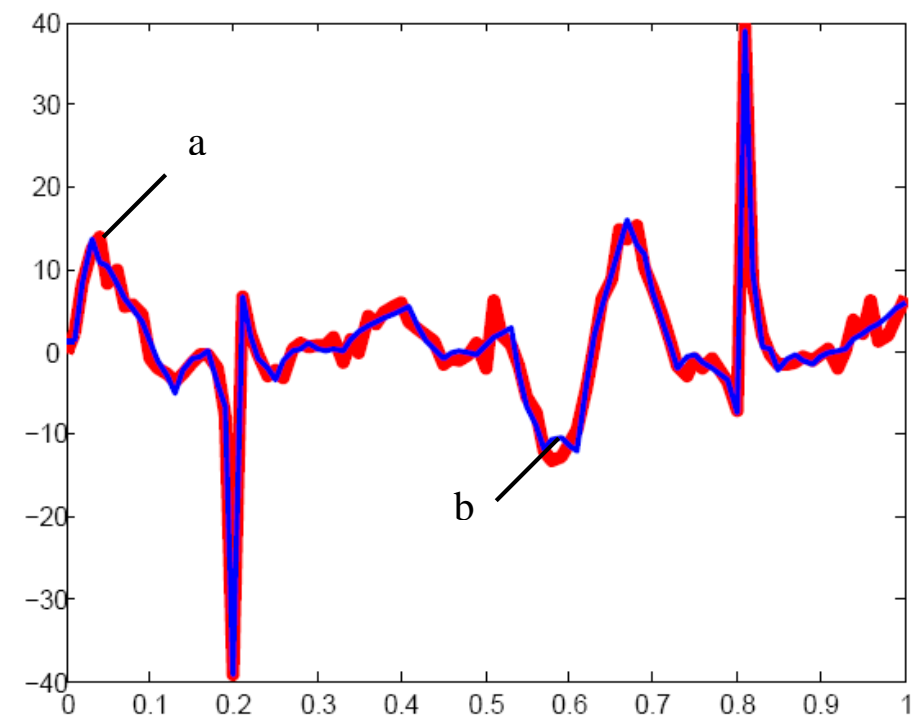

Fig. 4. Signal 2: noisy (red-a) and denoising (blue-b) result 
The results show that the proposed denoising algorithm can significantly decrease noises of technological acoustic and speech signals and increase the signal-to-noise ratio of technological acoustic and speech signals. The proposed algorithm has better performance than other algorithms on wavelet based reconstruction and distortion corrections, and has the advantages of fast computing speed and a wide range of adaptability.

\section{Conclusion}

In this paper, we proposed a new wavelet based denoising method which was developed by combining wavelet transform with reconstruction algorithm. This novel algorithm was applied to the noisy technological acoustic_and speech signals. The analysis was done by applying discrete wavelet transform to noisy technological acoustic and speech signals and applying new denoising method, then reconstruction of wavelets. The analysis was verified through simulation studies using MATLAB software. The proposed algorithm has better performance than other algorithms on wavelet based reconstruction and distortion corrections, and has the advantages of fast computing speed and a wide range of adaptability.

\section{Acknowledgements}

The article was supported by the State Oil Fund of Azerbaijan Republic in 2017 in the frame of grant project.

\section{References}

1. Pashayev F.H. Smoothing algorithms for characteristics of seismic-acoustic signals // Azerbaijan Oil Industry, 2013, №6, pp.42-48.

2. Pashayev F.H., Pakdel M., Rzaeva N. A Wavelet Based Denoising of Seismic Acoustic Signals / IV ALL-Ukranian Scientific-Practical conf. "Informatics and Systems sciences", Poltava, 2013, pp. 310-313.

3. Guluev G., Pashayev F. Pakdel M., Sattarova U. Prediction of Signal Characteristics Using Autoregressive Moving Average Method / IV International Conf. "Problems of Cybernetics and Informatics", vol.II, 12-14.09.2012, Baku, pp.102-104.

4. Sukhostat L.V. Adaptive noise reduction method based on empirical wavelet transform // Problems of Information Technology, 2017, №1, pp 53-58.

5. Lockwood O.G., Kanamori H. Wavelet analysis of the seismograms of the 2004 SumatraAndaman earthquake and its application to tsunami early warning // Geochemistry Geophysics Geosystems, 2006, no.7, pp. 1-10.

6. Pramanik N., Rabindra K. PAdvPSanda and Adarsh Singh. Daily river flow forecasting using wavelet ANN hybrid models // Journal of Hydroinformatics, 2011, no.1, pp. 49-63.

7. Haltmeier M. et al. Compressed sensing and sparsity in photoacoustic tomography // Journal of Optics, 2016, 18 114004, pp. 1-12.

8. Daubechies I., Ten Lectures on Wavelets, New York: SIAM, 1992, 357 p.

9. Mallat S.G. A Wavelet Tour of Signal Processing, Academic Press, 3rd Edition, 2008, 832 p.

10. Mallat S.G. A Theory of Multiresolution Signal Decomposition: The Wavelet Representation // IEEE Transactions on Pattern Analysis and Machine Intelligence, July 1989, 11(7), pp. 674-693.

11. Vidakovic B., Lozoya C.B. On time-dependent wavelet denoising // IEEE Transaction on Signal Processing, 1998, vol.46, no.9, pp. 2549-2554. 